\section{Über den Tellerrand}

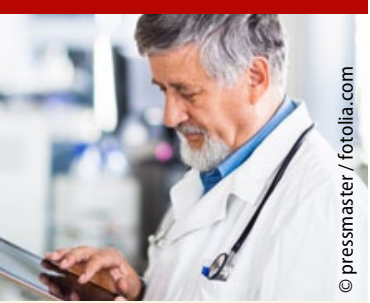

- Orthopädie \& Rheuma

Bei der Zeitschrift Orthopädie \& Rheuma steht neben abrechnungstechnischen, wirtschaftlich-rechtlichen und berufspolitischen Fragen die medizinische Fortbildung in praxisrelevanter Orthopädie, moderner Rheumatologie und angewandter Schmerztherapie im Mittelpunkt.

Die Zeitschrift ist offizielles Organ der Interdisziplinären Gesellschaft für orthopädische, unfallchirurgische und allgemeine Schmerztherapie e.V. (IGOST).

Aktuell können Sie lesen:

- Höhl R (2015) Praxischefs sollten auch mal loben. Orthopädie \& Rheuma 17:47

- Bublak R (2015) Operation offener Frakturen: Nicht in allen Fällen drängt die Zeit. Orthopädie \& Rheuma 17:10

- Krapf P (2015) RückenschmerzPatient erkrankt akut an einem Infekt. Orthopädie \& Rheuma 17:62

Sie finden diesen und weitere Beiträge über die Suchfunktion auf www.springermedizin.de

Als Abonnent von Springer Medizin e.Med können Sie alle Beiträge online im Volltext lesen.

- Springer Medizin e.Med bietet Ihnen:

- Online-Zugriff auf über 600 Fachzeitschriften

- 500 CME-Beiträge für Ihre Fortbildung

- auf Wunsch eine gedruckte Fachzeitschrift

Weitere Infos und das kostenlose Testangebot finden Sie unter www.springermedizin.de/eMed

Oper Orthop Traumatol 2015 .

27:191

DOI 10.1007/s00113-015-2744-2

(c) Springer-Verlag

Berlin Heidelberg 2015

Die Herausgeber der Zeitschrift übernehmen keine Verantwortung für diese Rubrik.

\title{
Neuartige Prothese
}

\section{Mit bionischen Händen wieder zupacken}

Chirurgen aus Österreich haben erstmals bei drei Männern mit Plexus-brachialis-Verletzung eine „bionische Rekonstruktion" der Hand gewagt. Verpflanzte Muskeln dienen als Verstärker, um eine Prothese zu steuern.

Einer der drei Männer ist vor vier Jahren beim Klettern abgestürzt, bei den anderen beiden hat ein Motorradunfall vor 19 und 14 Jahren das Nervengeflecht zerfetzt, das die Verbindung zwischen Hand und Rückenmark herstellt. Rekonstruktionsversuche bei den drei Patienten hatten lediglich bewirkt, dass sie wieder ihre Schulter und den Ellbogen etwas bewegen konnten, nicht aber die Hand, deren Innervation zunehmend verkümmerte. Aus diesem Grund haben Chirurgen um Professor Oskar Aszmann von der Universität Wien und Reha-Ingenieure von der Universität Göttingen einen anderen Ansatz versucht: Sie amputierten den drei Männern die Hand und passten ihnen sechs Wochen danach eine motorisierte Prothese an, die über noch vorhandene Nervenverbindungen gesteuert wird. Die drei Männer mussten zuvor ein mehrere Monate dauerndes kognitives Feedback-Training absolvieren, um die Muskeln zu trainieren, von denen später die Steuerungssignale für die Prothese abgegriffen wurden. Waren diesen Trockenübungen erfolgreich, amputierten die Wiener Chirurgen die Extremität etwa 10 bis $15 \mathrm{~cm}$ vom Handgelenk aufwärts.

Drei Monate nach der Amputation waren die Männer erstmals seit ihrem Unfall wieder in der Lage, beidhändig zuzupacken: Einen Ball aufheben ist mit der mechatronischen Kunsthand ebenso wenig ein Problem wie Hemden zuknöpfen, auch können sie damit ihre Haustür aufschließen, Kaffee einschenken oder Gemüse fürs Mittagessen kleinschneiden. Zudem spürten die Betroffenen kaum Phantomschmerzen. Durch die bionische Rekonstruktion wird das Phantom offenbar durch die wiedergewonnene Funktionalität der Hand ersetzt, so Aszmann.

www.springermedizin.de

Unter „Medizin aktuell“ finden Sie weitere aktuelle Beiträge aus Ihrem Fachgebiet: www.springermedizin.de/ medizin

\section{Online miträtseln: Blickdiagnose interaktiv Schmerzhafte Dellen in der Ferse}

Ein 35-jähriger Patient, der in seiner Freizeit regelmäßig Squash spielt, klagt über seit mehreren Monaten brennende, stechende Schmerzen an den Fußsohlen beim Gehen, die bei Entlastung der Füße nachlassen. An den Fersen beidseits findet sich eine weißlich verfärbte, aufgequollen wirkende Hornhaut mit flachen, wie ausgestanzt wirkenden, teilweise konfluierenden Vertiefungen. Seit der Jugend bestehe vermehrte Schwitzneigung an den Fußsohlen.

Was ist Ihre Diagnose?

- Erythrasma

- Tinea pedis

- Petechien

Keratoma sulcatum

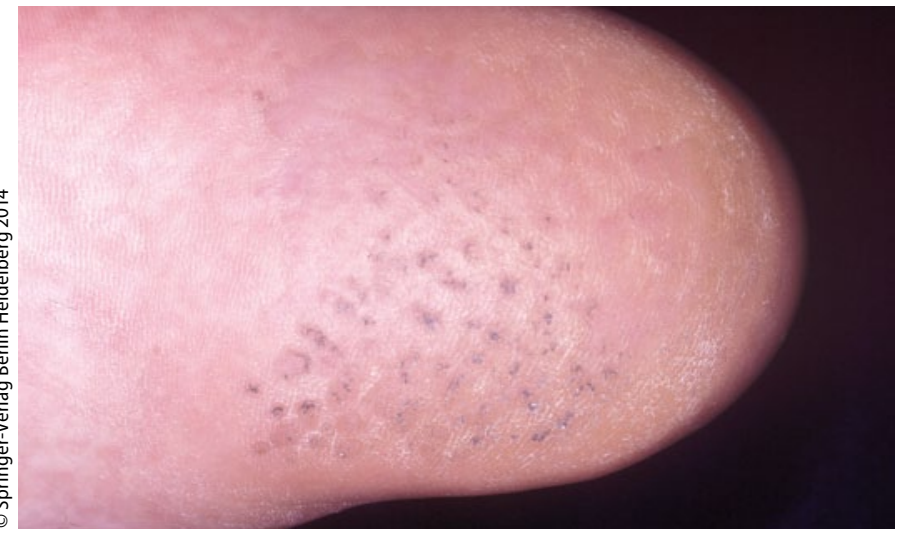

A Warum schmerzen die Fußsohlen beim Gehen?

Lösung

Auf www.springermedizin. de/5195782 erfahren Sie sofort, ob Sie richtig liegen - nebst interessanten Infos zu diesem Fall.
Rätseln Sie weiter unter:

- www.springermedizin.de/ blickdiagnose-interaktiv 